\title{
Efeito do isostretching na resistência muscular de abdominais, glúteo máximo e extensores de tronco, incapacidade e dor em pacientes com lombalgia
}

\section{The Isostretching effect in the muscle strength of gluteus maximus, abdominal and the trunk extensor, incapacity and pain in patients with low back pain}

\author{
Christiane de Souza Guerino Macedo ${ }^{[a]}$, Polyana Cortizo Debiagi ${ }^{[\mathrm{b}]}$, \\ Fernanda Marçola de Andrade ${ }^{[c]}$
}

[a] Docente do Departamento de Fisioterapia da Universidade Estadual de Londrina, Mestre em Biodinâmica do Movimento Humano (USP/SP), Coordenadora do Projeto de Extensão Universitária (UEL), Londrina, PR - Brasil, e-mail: chmacedouel@yahoo.com.br

[b] Discente do Curso de Graduação em Fisioterapia da Universidade Estadual de Londrina, Projeto de Extensão Universitária (UEL), Londrina, PR - Brasil, e-mail: polyanacb@yahoo.com.br

[c] Discente do Curso de Graduação em Fisioterapia da Universidade Estadual de Londrina, Projeto de Extensão Universitária (UEL), Londrina, PR - Brasil, e-mail: fermarsola@yahoo.com.br

\section{Resumo}

Objetivou-se determinar a influência do método Isostretching na resistência muscular do glúteo máximo, abdominais e extensores de tronco, incapacidade e dor de pacientes com lombalgia. Material e métodos: Este estudo caracterizou-se como um ensaio clínico aleatório, constituído de 18 mulheres, entre 18 e 25 anos, com dor cinco ou mais na escala visual análoga (EVA) na região lombar, no mínimo três vezes por semana, randomizadas em grupo ativo e controle. Foram excluídas as submetidas a tratamentos fisioterápicos ou que tenham passado por cirurgia recente e com outras patologias. As pacientes foram avaliadas (avaliadores cegos) no início, após 10 e 20 sessões, pelo questionário Roland-Morris, EVA e Teste de Repetição Máxima em um minuto para abdominais, extensores de tronco e glúteo máximo. Para a análise estatística, foi aplicado o teste de Shapiro Wilk, Teste t de Student e ANOVA. Resultados: A comparação entre os valores iniciais e finais no grupo ativo para a análise da resistência dos músculos abdominais, glúteo máximo, extensores de tronco, incapacidade e dor apresentou $\mathrm{p}=0,00$ e no grupo controle $\mathrm{p}=0,02$ para o glúteo máximo. A análise dos três momentos de avaliação no grupo ativo evidenciou para a resistência dos músculos abdominais, glúteo máximo e dor $\mathrm{p}=0,00$, incapacidade $\mathrm{p}=0,01$ e para os extensores de tronco $\mathrm{p}$ $=0,80$ e o grupo controle não evidenciou alteração. Conclusão: O Isostretching mostrou-se eficiente para diminuir a incapacidade e dor, bem como para o aumento da resistência muscular de abdominais, glúteo máximo e extensores de tronco de pacientes com lombalgia.

Palavras-chave: Isostretching. Resistência muscular. Lombalgia. Fisioterapia. 


\begin{abstract}
To determine how the muscle strength of gluteus maximus, abdominal and trunk extensor, the incapacity and pain in patients with low back pain could be influenced by Isostretching method. Methods: This work consisted on a random clinical trial approach where 18 women were divided into active and control group. Requirements used to make a participant eligible were 18-25 age and at least 5 low back pain by Visual Analogical Scale (VAS) three times a week. Those who subjected to other physiotherapy treatments, recently surgery or other pathology were excluded. The assessments were performed at the beginning, after 10 and 20 sessions by two blind raters, using the Roland Morris'questionnaires, the VAS and the Repetition Maximum Test in one minute. The Shapiro Wilk test, Student's t-test and the ANOVA were used for the statistical analysis. Results: The comparison between initial and final values in the active group to analyze the muscle strength of abdominal, gluteus maximus, the trunk extensors, disability and pain showed $p=0,00$ and in the control group $p=0,02$ for the gluteus maximus. The analysis of the three stages of assessment for active group showed for the muscle strength of abdominal, gluteus maximus and pain $p=0,00$, incapacity $p=0,01$ and the extensors of trunk $p=0,80$. The control group didn't show any change. Conclusions: The Isostretching is efficient to reduce the pain and disability, and to increase the muscle strength of abdominal, gluteus maximus and the trunk extensor of patients with low back pain.
\end{abstract}

Keywords: Isostretching. Muscle strength. Low back pain. Physiotherapy.

\title{
Introdução
}

Lombalgia são todas as condições de dor, com ou sem rigidez, localizadas na região inferior do dorso, em uma área situada entre o último arco costal e a prega glútea (1). Nos Estados Unidos, a lombalgia é a causa mais comum de limitação de atividades entre as pessoas com menos de 45 anos e a segunda mais frequente para visitas médicas. No Brasil, cerca de 10 milhões de brasileiros ficam incapacitados por causa desta morbidade e pelo menos $70 \%$ da população sofrerá um episódio de dor na vida (2).

A dor lombar apresenta etiologia multifatorial, incluindo fatores socioeconômicos e demográficos, estilo de vida urbano sedentário, obesidade, fumo, posturas viciosas durante o trabalho, aumento da sobrevida média da população e outros (3). Dentre os distúrbios dolorosos, a dor lombar constitui causa frequente de morbidade e incapacidade, sendo ultrapassada somente pela cefaleia (4).

A incapacidade de estabilização da coluna vertebral causada pelo desequilíbrio entre a função dos músculos extensores e flexores do tronco é forte indício para o desenvolvimento de distúrbios da coluna lombar. A dor lombar acontece em consequência de um comprometimento da força ou resistência isométrica de músculos desse segmento. Atualmente, existem evidências que sugerem a inclusão de exercícios voltados para o fortalecimento dos músculos envolvidos na flexão e extensão do tronco nos programas de prevenção e reabilitação da dor na região da coluna lombar (5).

Sabe-se da existência de uma rápida atrofia dos músculos eretores da espinha após um episódio de dor lombar, a qual permanece mesmo após a regressão dos sintomas (6). Este quadro é reversível e a recidiva da dor é reduzida quando realizados exercícios de força e resistência isométrica direcionados a esses músculos. Afirma-se que a hipotonicidade (por causa do desuso), a permanência prolongada em determinadas posições ou mesmo a fadiga por movimento repetitivo, causam transferência excessiva de carga, resultando em dor (7).

Para os movimentos do tronco e manutenção adequada da postura, há necessidade de ações musculares seletivas e compatíveis com a exigência das atividades diárias. Os músculos eretores asseguram o posicionamento correto do tronco na posição ereta ${ }^{7}$ e seu déficit de desempenho induz à instabilidade da coluna, contribuindo para o desenvolvimento de processos álgicos e fadiga muscular 
(8). Os músculos abdominais auxiliam na manutenção do equilíbrio e atuam diretamente na estática e na dinâmica da pelve, sendo assim de grande importância para a postura do corpo. A fraqueza leve ou moderada do músculo glúteo máximo, em conjunto com a dos isquiotibiais, permite que a pelve se incline para frente, acentuando a lordose.

$\mathrm{Na}$ presença dessas alterações biomecânicas, as chances de se desenvolver uma lombalgia aumentam (9). Portanto, para o tratamento, prevenção ou reabilitação deve ser realizado um programa de reequilíbrio corporal, com base em flexibilidade, fortalecimento muscular e adequações das atividades de vida diária.

Dentre os métodos de abordagem global do paciente, pode-se citar o Isostretching. Segundo Redondo (10), esse método se baseia em exercícios que focam o alongamento e fortalecimento corporal. É um método postural porque os exercícios são realizados na posição correta da coluna, respeitando suas curvas fisiológicas. Global, porque todo o corpo é trabalhado a cada exercício, pois contração e alongamento são incluídos em cada postura. Ereto, porque há o trabalho da musculatura paravertebral na solicitação do autocrescimento. O método trabalha, ao mesmo tempo, o fortalecimento isométrico (abdominais, glúteos, flexores de quadril e assoalho pélvico), o alongamento global, autocrescimento, a respiração e o posicionamento adequado entre o quadril e a coluna vertebral. Assim, estimula a coordenação a propriocepção e a percepção corporal do paciente (11).

O objetivo deste trabalho foi determinar a influência do método Isostretching na resistência muscular do glúteo máximo, abdominais e extensores de tronco dos pacientes após 10 e 20 sessões, assim como na incapacidade e diminuição da dor lombar.

\section{Material e métodos}

Neste estudo, caracterizado como um ensaio clínico aleatório, participaram 18 pacientes, randomizados, por envelopes pardos, em grupo ativo (nove) e grupo controle (nove), dos quais três não compareceram às reavaliações periódicas, assim composto por seis indivíduos. Todos os participantes eram do gênero feminino, com idade entre 18 e 25 anos, apresentando dor cinco ou mais, pela escala visual análoga (EVA), na região lombar, no mínimo três vezes na semana. Foram excluídos aqueles que recebiam outros tipos de tratamento fisioterápico, submetidos à cirurgia recentemente ou que apresentaram outras patologias associadas (reumáticas, hérnia de disco, fraturas, artrose, dor irradiada para membros, entre outras).

As avaliações foram realizadas, por dois avaliadores cegos, em três tempos (início, após as dez primeiras sessões e após 20 sessões). Foi aplicado o questionário Roland-Morris, a Escala Visual Analógica (EVA) e o Teste de Repetição Máxima em um minuto para os abdominais, glúteo máximo e extensores de tronco. O questionário Roland-Morris (12) e a Escala Visual Analógica (EVA) são amplamente utilizados em pesquisas com lombalgia (13).

Para a realização do Teste de Repetição Máxima em um minuto (RM) para os abdominais, a paciente encontrava-se em decúbito dorsal, com os membros inferiores fletidos, membros superiores cruzados sobre o tronco, sendo estabilizada pelo avaliador nos pés e joelhos, realizando flexão anterior de tronco até encostar os braços nos joelhos.

Para a análise dos extensores de tronco, a paciente foi posicionada em decúbito ventral, com as mãos atrás da cabeça e com um apoio na região da testa $(10 \mathrm{~cm}$ de altura), sendo estabilizada pelo avaliador na região posterior da pelve. Realizou, primeiramente, uma extensão máxima de tronco, onde foi marcada a altura, e após breve descanso iniciou o teste, no qual deveria atingir a altura previamente marcada.

A avaliação do glúteo máximo foi desenvolvida com a paciente ajoelhada (flexão do joelho de $90^{\circ}$ ) em um apoio ( $40 \mathrm{~cm}$ de altura), e com $90^{\circ}$ de flexão de tronco sobre uma maca, com uma caneleira de $3 \mathrm{~kg}$ no membro inferior dominante. Realizou, primeiramente, uma extensão máxima de quadril, onde era marcada a altura e, após um breve descanso, foi iniciado o teste atingindo sempre a marcação inicial.

Todas as repetições só foram consideradas quando realizadas por completo, ou seja, atingia os joelhos, no caso dos abdominais, e a altura máxima previamente estabelecida, no caso do glúteo máximo e extensores de tronco, sempre retornando ao apoio inicial. 
Tanto o grupo ativo como o controle foram informados sobre os procedimentos utilizados, estando assim de acordo em participar do estudo de forma voluntária, assinando um Termo de Consentimento Livre e Esclarecido. O grupo controle se comprometeu a não realizar nenhum tipo de tratamento durante o período do estudo.

O grupo ativo participou de um programa de tratamento constituído de exercícios do método Isostretching. Foram realizadas 20 sessões (com uma reavaliação após as dez primeiras sessões e outra após o término do tratamento), com duração média de 50 minutos. Na primeira delas, os pacientes receberam informações básicas sobre o método: expiração profunda prolongada, autocrescimento e contração isométrica de glúteos, quadríceps, abdominais e assoalho pélvico. As sessões foram realizadas de duas a três vezes por semana, utilizando-se somente colchonetes. Os exercícios realizados foram divididos nas posições deitado, sentado e em pé, totalizando nove exercícios por sessão. Cada um desses exercícios foram repetidos nove vezes, com intervalo a cada três repetições.

Para a análise estatística foi calculada a distribuição de normalidade das variáveis pelo teste de Shapiro Wilk. Em função das variáveis se apresentarem com distribuição normal, foi aplicado o teste t de Student para a comparação dos valores da primeira e última avaliação e o ANOVA (para os três momentos de coleta de dados). Calculou-se o tamanho do efeito para os dois grupos, sendo também comparado pelo teste t de Student. O nível de significância estatística foi estabelecido em 5\% e foi utilizado o programa SPSS, versão 13.0. O estudo foi aprovado pelo Comitê de Ética em Pesquisa da Universidade Estadual de Londrina (Parecer 056/08).

\section{Resultado}

A amostra foi composta por pacientes com lombalgia, todas do gênero feminino, com idade média de 20,9 (DP = 1,62). O grupo ativo apresentou idade de 21,11(DP = 2,02) e o controle de 20,6 $(\mathrm{DP}=0,81)$, sedentárias e estudantes de nível superior. A análise estatística para o parâmetro idade apresentou $\mathrm{p}=0,31$, caracterizando a amostra como homogênea em relação à idade.

Os resultados obtidos na análise das variáveis nos três momentos (pré-tratamento, após 10 sessões e ao término de 30 sessões) para o grupo ativo e controle estão apresentados na Tabela 1.

Tabela 1 - Resultados apontados para os grupos ativo e controle

\begin{tabular}{|c|c|c|c|}
\hline & & $\begin{array}{c}\text { Avaliação após } 10 \\
\text { sessões }\end{array}$ & Avaliação final \\
\hline EVA (grupo ativo) & $5,88(\mathrm{DP}=1,27)$ & $2,22(\mathrm{DP}=1,09)$ & $5,44(\mathrm{DP}=2,24)$ \\
\hline EVA (grupo controle) & $6,17(\mathrm{DP}=0,75)$ & $4,67(\mathrm{DP}=1,97)$ & $5(\mathrm{DP}=1,90)$ \\
\hline Roland Morris (grupo ativo) & $5,44(\mathrm{DP}=2,24)$ & $3,33(\mathrm{DP}=2,23)$ & $2,44(\mathrm{DP}=1,51)$ \\
\hline Roland Morris (grupo controle) & $4,5(\mathrm{DP}=1,87)$ & $3,33(\mathrm{DP}=1,97)$ & $4,83(\mathrm{DP}=1,83)$ \\
\hline $\begin{array}{l}\text { Número de repetições de exercícios } \\
\text { Abdominais (grupo ativo) }\end{array}$ & $21,44(\mathrm{DP}=3,28)$ & $27,11(\mathrm{DP}=3,55)$ & $29,78(\mathrm{DP}=4,57)$ \\
\hline $\begin{array}{l}\text { Número de repetições de exercícios } \\
\text { Abdominais (grupo controle) }\end{array}$ & $27,67(\mathrm{DP}=6,53)$ & $26,67(\mathrm{DP}=6,12)$ & $26,67(\mathrm{DP}=6,62)$ \\
\hline $\begin{array}{l}\text { Número de repetições de exercícios } \\
\text { para Extensores do Tronco (grupo } \\
\text { ativo) }\end{array}$ & $34,78(\mathrm{DP}=21,39)$ & $47,33(\mathrm{DP}=18,15)$ & $54,89(\mathrm{DP}=14,29)$ \\
\hline $\begin{array}{l}\text { Número de repetições de exercícios } \\
\text { para Extensores do Tronco (grupo } \\
\text { controle) }\end{array}$ & $46(\mathrm{DP}=6,81)$ & $43,33(\mathrm{DP}=8,52)$ & $43,67(\mathrm{DP}=9,05)$ \\
\hline $\begin{array}{l}\text { Número de repetições de exercícios } \\
\text { para Glúteo (grupo ativo) }\end{array}$ & $34,11(\mathrm{DP}=8,03)$ & $44,33(\mathrm{DP}=5,87)$ & $50,33(\mathrm{DP}=4,06)$ \\
\hline $\begin{array}{l}\text { Número de repetições de exercícios } \\
\text { para Glúteo (grupo controle) }\end{array}$ & $37,5(\mathrm{DP}=10,27)$ & $39,17(\mathrm{DP}=10,09)$ & $38,67(\mathrm{DP}=10,31)$ \\
\hline
\end{tabular}


A comparação dos valores iniciais e finais (após 30 sessões) no grupo ativo apontou diferença significativa para as variáveis: EVA $(\mathrm{p}=0,00)$, índice do Questionário de Roland Morris $(\mathrm{p}$ $=0,00)$, número de repetições máxima em um minuto para músculos abdominias $(\mathrm{p}=0,00)$, extensores do tronco $(p=0,00)$ e glúteos $(p=0,00)$. A evolução dos valores durante os três momentos de coleta neste grupo apontou: EVA $(\mathrm{p}=0,00)$, índice do Questionário de Roland Morris $(\mathrm{p}=0,01)$, número de repetições máxima em um minuto para músculos abdominias $(p=0,00)$, extensores do tronco $(p=0,08)$ e glúteos $(\mathrm{p}=0,00)$.

Os valores apresentados pelo grupo controle demonstraram diferença significativa na comparação da avaliação inicial e final somente para o número de repetições dos exercícios de glúteo $(\mathrm{p}=0,03)$. A análise da evolução nos três momentos de coleta não evidenciou diferença para nenhuma variável estudada.

A análise do tamanho do efeito entre os grupos apresentou para o parâmetro dor $\mathrm{p}=0,01$, para a incapacidade analisada pelo questionário Roland Morris $\mathrm{p}=0,03$, para a resistência do glúteo máximo $\mathrm{p}=0,02$, para os abdominais $\mathrm{p}=0,00$ e para os extensores do tronco $\mathrm{p}=0,03$.

\section{Discussão}

A amostra do presente estudo foi constituída por indivíduos do gênero feminino, estudantes de nível superior e sedentárias. Estudos comprovam que as mulheres possuem maior risco e maior severidade para diversas condições clínicas de dor por características anátomo-funcionais, em função da menor estatura, menor massa muscular e óssea, articulações mais frágeis e menos adaptáveis ao esforço físico pesado e maior peso de gordura; fatores que podem colaborar para o surgimento das dores lombares crônicas (14). No estudo de Mayworm (15), verificou-se que 61,5\% das mulheres participantes eram sedentárias e que a comparação entre aquelas que praticavam atividade física com as que não apresentavam dor lombar foi significante $(\mathrm{p}=0,00)$, denotando a importância da prática da atividade física como profilaxia da dor lombar.

A melhora da dor e diminuição da incapacidade física com o Isostretching já foi apresentada. Facci (16) avaliou 20 pacientes com quadro de lombalgia crônica utilizando a EVA, o questionário Roland Morris e o item "dor" do questionário de qualidade de vida SF-36, resultando na redução significativa das três variáveis $(\mathrm{p}=0,00)$. No presente estudo, confirmam-se os efeitos positivos do método, em função da melhora apresentada pelas pacientes. Em relação ao efeito do método Isostretching na melhora da incapacidade dos pacientes, pôde se observar que estes estão com menos restrições de movimento e atividades, apresentando maior disposição para as atividades de vida diária. Os itens mais citados no questionário Roland Morris e que apresentaram melhora durante o estudo foram em relação a deitar frequentemente para descansar, evitar atividades de abaixar e ajoelhar, apresentar dor quase o tempo todo e alterações no humor.

As propostas de reabilitação das disfunções da coluna lombar são amplamente apresentadas e discutidas na literatura. A necessidade da busca pelo reequilíbrio muscular e correção biomecânica é apontada como eixo principal para a boa evolução do paciente. Os exercícios são a forma mais segura e eficaz para melhorar a flexibilidade, força e função muscular, bem como reduzir a dor lombar tanto na prevenção como na reabilitação desses pacientes (17). Observa-se que programas de fortalecimento e estabilidade do tronco são efetivos na redução da dor lombar em mulheres (18).

A necessidade de utilização de condutas fisioterápicas que contemplem o trabalho de força e resistência muscular, como o método Isostretching utilizado no presente estudo, é justificada por vários estudos. Aponta-se que indivíduos com lombalgia entram em fadiga rapidamente por causa da baixa força muscular dos músculos flexores e extensores da coluna lombar (19). Indivíduos com disfunções lombares apresentam redução na resistência da musculatura paravertebral, tendo como possíveis causas alterações no padrão de controle motor e sobrecarga prolongada do sistema passivo de suporte (20). Também a baixa resistência à fadiga dos músculos paraespinhais é comum em pacientes com dor lombar crônica (21), que evitam, desse modo, movimentos do seu cotidiano por medo da dor e suas 
consequências. Assim, iniciam um ciclo no qual utilizam cada vez menos essa musculatura por causa de seu maior comprometimento, evoluindo para atrofia e dor (22).

Comprovando a importância da avaliação e reabilitação do músculo transverso do abdômen, estabelece-se que esse músculo é ativado, em indivíduos normais, antes de ser iniciado um movimento com os membros; entretanto, na presença da dor lombar, essa ativação é mais lenta. Também, que a ativação precoce do músculo transverso abdominal, como mecanismo de proteção para a coluna lombar, é perdida em pacientes com queixa de lombalgia (23).

May e Johnson (24) e Lewis, Morris, Walsh (25) estabelecem, em revisões sistemáticas, a existência de evidências de que exercícios de estabilização diminuem a dor e incapacidade de indivíduos com lombalgia crônica. Afirma-se que os exercícios de fortalecimento do tronco apresentam efetividade quando comparados ao grupo controle, sem exercícios; e também que a inclusão de exercícios mais intensos e motivacionais aumentam o efeito do tratamento (26). Ainda, exercícios de fortalecimento e exercícios aeróbios promovem o retorno ao trabalho em indivíduos com dor lombar crônica (27). Por outro lado, observa-se que exercícios tradicionais de fortalecimento dos músculos abdominais e extensores do tronco têm sido alvo de críticas por submeter a coluna vertebral a altas cargas de trabalho, aumentando o risco de nova lesão (28).

As estruturas inertes são interligadas, formando uma unidade funcional ligamentar que parece estar intimamente ligada à ação da musculatura do tronco por meio das inserções e envoltórios musculares, aumentando a rigidez tecidual e, consequentemente, aumentando a estabilidade (29). Acredita-se que os exercícios de estabilização, realizados de forma isométrica e sustentada, facilitem o equilíbrio muscular do complexo lombo-pelve-quadril e que esse tipo de conduta facilite a interação entre as estruturas anatômicas estáticas e dinâmicas na execução das atividades funcionais (30).

O método Isostretching, com o uso do autocrecimento, contração isométrica (abdominais, glúteos, quadríceps e assoalho pélvico), alongamento muscular, além de uma expiração lenta e prolongada, desenvolve as capacidades musculares em desequilíbrio. Dessa forma, o protocolo desenvolvido neste estudo mostrou-se eficaz no desenvolvimento da resistência muscular, melhora da incapacidade e diminuição da dor dos pacientes analisados.

\section{Conclusão}

Concluiu-se que o método Isostretching mostrou-se eficiente para diminuir a incapacidade e dor, bem como aumentar a resistência muscular de abdominais, glúteo máximo e extensores de tronco nos pacientes com lombalgia. Ainda, estabelece-se a importância de trabalhos de força e resistência muscular para indivíduos com dor lombar crônica.

\section{Referências}

1. Pereira MJ, Mendes CR, Batista RSB, Ferreira BL. Efeitos do método pilates na lombalgia: estudo de 2 casos. 2008. [acesso 10 dez. 2008]. Disponível em: www.wgate.com.br

2. Khouri ME, Corbett CEP, Cordeiro Q. Classificação neurofisiológica da Síndrome do Túnel do CarpoPrevalência de lombalgia em garimpeiros de Serra Pelada, Pará/Brasil. Acta Fisiátrica. 2008;15(2):82-6.

3. Matos GM, Hennington EA, Hoefel AL, Costa JSD. Dor lombar em usuários de um plano de saúde: prevalência e fatores associados. Cad Saúde Pública. 2008;24(9): 2115-22.

4. Abreu AM, Faria CDCM, Cardoso SMV, Salmela LFT. Versão brasileira do Fear Avoidance Beliefs Questionnaire. Cad Saúde Pública. 2008;24(3):615-23. 
5. Aguilar GK, Sampaio VL, Sandoval RA. Análise da força lombar através da balança analógica adaptada. Revista Digital. 2007;Ano 12(115). [acesso 15 dez. 2008]. Disponível em: www.efdeportes.com

6. Gonçalves M, Barbosa FSS. Análise de parâmetros de força e resistência dos músculos eretores da espinha lombar durante a realização de exercício isométrico em diferentes níveis de esforço. Rev Bras Med Espote. 2005;11(2):109-14.

7. Costa D, Palma A. O efeito do treinamento contra resistência da síndrome da dor lombar. Rev Port Cien Desp. 2005;5(2):224-34.

8. Barbosa FSS, Gonçalves M. Comparação entre protocolos de exaustão e de 30 segundos utilizados na avaliação da fadiga eletromiográfica dos músculos eretores da espinha. Rev Bras Fisioter. 2005;9(1):77-83.

9. Kendall FP, McCreary EK, Provance PG. Músculos provas e funções. 5a ed. São Paulo: Manole; 2007.

10. Redondo B. Isostretching: a ginástica da coluna, Piracicaba: Skin Direct Store; 2001.

11. Macedo CSG. Isostretching como prevenção e tratamento das lesões desportivas, 2006. [acesso 13 dez. 2008]. Disponível em: www.fisioesporte.com.br

12. Nusbaum L, Natour J, Ferraz MB, Goldenberg J. Translation, adaptation and validation of the RolandMorris questionnaire - Brazil Roland-Morris. Braz J Med Biol Res. 2001;34(2):203-10.

13. Roland M, Fairbank J. The Roland-Morris disability questionnaire and the oswestry disability questionnaire. Spine Philadelphia PA. 2000;25(24):3115-24.

14. Breder VF, Oliveira DF, Dantas EHM, Silva MAG. Prevalência de lombalgia em motoristas de ônibus urbano. Fisioterapia Brasil. 2006;7(4):290-4.

15. Mayworm SH, Pereira JS, Silva MAG. Prevalência de lombalgia em costureiras de moda íntima em Nova Friburgo, RJ. Fisioterapia Brasil. 2008;9(3):329-34.

16. Facci LMO. Isostretching no tratamento da lombalgia crônica. Fisioterapia Brasil. 2006;7(2):245-61.

17. Byrne K, Doody C, Hurley DA. Exercise therapy for low back pain: a small-scale exploratory survey of current physiotherapy practice in the republic of Ireland acute hospital setting. Man Ther. 2006;11(4):272-8.

18. Carpes FP, Reinehr FB, Mota CB. Effects of a program for trunk strength and stability on pain, low back and pelvis kinematics, and body balance: a pilot study. J Bodyw Mov Ther. 2008;12(1):22-30.

19. Hart JM, Fritz JM, Kerrigan DC, Saliba EN, Gansneder BM, Ingersoll CD. Reduced quadriceps activation after lumbar paraspinal fatiguing exercise. J Athl Train. 2006;41(1):79-86.

20. O’Sullivan PB, Mitchell T, Bulich P, Waller R, Holte J. The relationship between posture and back muscle endurance in industrial workers with flexion-related back pain. Man Ther. 2006;11(4):264-71.

21. Srinivasan J, Balasubramanian V. Low back pain and muscle fatigue due to road cycling-An sEMG study. J Bodywork MovTherap. 2007;11(3):260-6.

22. Kawano MM, Souza RB, Oliveira BIR, Menacho MO, Cardoso APRG, Nakamura FY, et al. Comparação da fadiga eletromiográfica dos músculos paraespinhais e da cinemática angular da coluna entre indivíduos com e sem dor lombar. Rev Bras Med Esporte. 2008;14(3):209-14.

23. Potts AMA, Morrissey MC, Critchley D. The response of the transverse abdominis and internal oblique muscles to different postures. Man Ther. 2006;11(1):54-60.

24. May S, Johnson R. Stabilisation exercises for low back pain: a systematic review. Physiotherapy. 2008;94:179-89. 
25. Lewis A, Morris ME, Walsh C. Are physiotherapy exercises effective in reducing chronic low back pain? Physical Therapy Reviews. 2008;13(1):37-44.

26. Slade SC, Keating JL. Trunk-strengthening exercises for chronic low back pain: a systematic review. J Manipulative Physiol Ther. 2006;29(2):163-73

27. Hettinga DM, Jackson A, Moffett JK, May S, Mercer C, Woby SR. A systematic review and synthesis of higher quality evidence of the effectiveness of exercise interventions for non-specific low back pain of at least 6 weeks' duration. Physical Therapy Reviews. 2007;12(3):221-32.

28. França FJR, Burke TN, Claret DC, Marques AP. Estabilização segmentar da coluna lombar nas lombalgias: uma revisão bibliográfica e um programa de exercícios. Fisioterapia e Pesquisa. 2008;15(2):200-6.

29. Teixeira-Salmela LF, Sakamoto ACL, Siqueira FB. Mecanismos de estabilização da coluna lombar: uma revisão da literatura. Fisioter Mov. 2004;17(4):51-8.

30. Tibúrcio NS, Sales TB, Freitas DG, Andrade RM. O impacto dos exercícios de estabilização central na qualidade de vida de pacientes com lombalgia crônica. Terapia Manual. 2008;6(26):229-34.

Recebido: 26/01/2009

Received: 01/26/2009

Aprovado: 02/10/2009

Approved: 10/02/2009 